\title{
SURFACE ELASTICITY FOR APPLICATIONS TO MATERIAL MODELLING AT SMALL SCALES
}

\section{LEONID A. IGUMNOV', VICTOR A. EREMEYEV ${ }^{2}$, ALEKSANDR A. IPATOV ${ }^{3}$ AND ANATOLY M. BRAGOV ${ }^{4}$}

\author{
${ }^{1}$ Research Institute for Mechanics, \\ National Research Lobachevsky State University of Nizhny Novgorod, \\ Gagarin Avenue 23, Nizhny Novgorod, 603950, Russia \\ igumnov@mech.unn.ru, http://eng.unn.ru/ \\ ${ }^{2}$ Research Institute for Mechanics, \\ National Research Lobachevsky State University of Nizhny Novgorod, \\ Gagarin Avenue 23, Nizhny Novgorod, 603950, Russia \\ Gdańsk University of Technology, \\ ul. Gabriela Narutowicza 11/12, 80-233 Gdańsk, Poland \\ Dipartimento di Ingegneria Civile, Ambientale e Architettura \\ Università degli Studi di Cagliari \\ Via Marengo 2, 09123 Cagliari, Italy \\ vicereme@pg.edu.pl, victor.eremeev@unica.it, https://wilis.pg.edu.pl/kwm \\ ${ }^{3}$ Research Institute for Mechanics, \\ National Research Lobachevsky State University of Nizhny Novgorod, \\ Gagarin Avenue 23, Nizhny Novgorod, 603950, Russia \\ ipatov@mech.unn.ru, http://eng.unn.ru/ \\ ${ }^{4}$ Research Institute for Mechanics, \\ National Research Lobachevsky State University of Nizhny Novgorod, \\ Gagarin Avenue 23, Nizhny Novgorod, 603950, Russia \\ bragov@mech.unn.ru, http://eng.unn.ru/
}

Key words: Surface elasticity, Effective properties, Surface stresses.

\begin{abstract}
We discuss the various applications of the surface elasticity to determination of effective properties of materials and some related phenomena as surface wave propagation. In the frame of surface elasticity in addition to the constitutive relations in the bulk we independently introduce constitutive relations at the surface. Nowadays the most popular models of surface elasticity relates to the models by Gurtin and Murdoch and by Steigmann and Ogden. First we discuss some useful surface elasticity models. The corresponding boundary dynamic boundary conditions are derived at the smooth parts of the boundary as well as at edges and corner points. Let us underline that these conditions include also dynamic terms. As a result, we have here a dynamic generalization of the Laplace-Young equation known from the theory of capillarity. Second, we discuss the influence of the surface stresses
\end{abstract}


at the effective stiffness parameters of layered plates and shallow shells. For small deformations we derived the exact formulae for modified tangent and bending stiffness parameters of the plates and shells. The influence of residual surface stresses is also discussed. Unlike to previous case where surface stresses are slightly changing the material properties, there is another example of essential influence of surface properties. This example relates to the propagation of anti-plane surface waves. We discuss some peculiarities of the wave propagation.

\section{INTRODUCTION}

Nowadays grows the interest to modeling of material behavior at small scales based on advances in nanotechnologies. It is well-established that the material behavior at small scales is different from one observed at the macro-scale. In particular, various size-effects can be observed at the nanoscale, which relate to high surface-to-volume ration in comparison with a characteristic size [1]. In order to capture these phenomena various enhancements of continuum models were proposed including the surface elasticity. The model of surface elasticity is based on introduction of surface energy and surface stresses independently on the constitutive equations in the bulk. Nowadays the most popular models of surface elasticity were proposed by Gurtin and Murdoch [2, 3] and by Steigmann and Ogden [4, 5]. Some other models are also known in the literature, which can describe surface/interface related phenomena, see, e.g., [1, 6-8] and the reference therein. From the physical point of view the surface elasticity describes coupled deformations of an elastic se its surface or its part a surface structures can
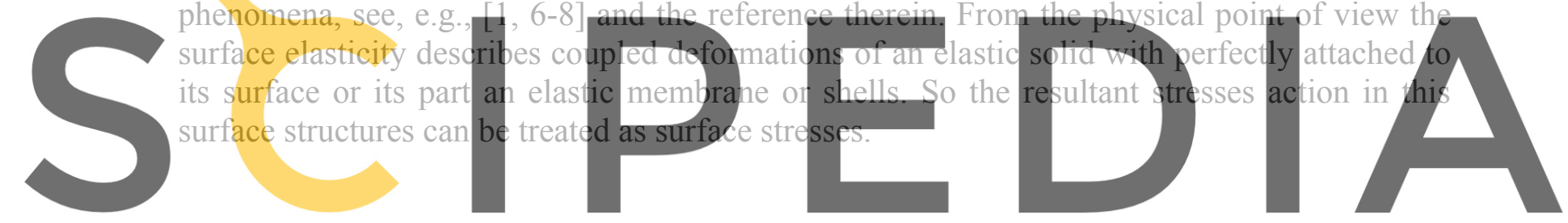

2 SURFACE ELASTICITY

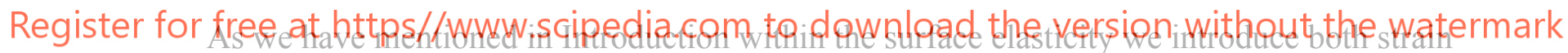

energy $W$ in the bulk and the surface strain energy $U$. In the case of infinitesimal deformations

of isotropic solids the simplest model of the surface elasticity is the linear Gurtin-Mivurdoch model based on the constitutive equation [8]

$$
U=\mu_{s} \boldsymbol{e}: \boldsymbol{e}+1 / 2 \lambda_{s} t r^{2} \boldsymbol{e},
$$

where $\boldsymbol{e}$ is the surface strain tensor and $\mu_{s}$ and $\lambda_{s}$ are surface Lamé moduli. Eq. (1) is a surface analogue of the Hooke's law in the bulk.

\subsection{Effective properties}

A typical dependence of a material property $P$ at the nanoscale can be presented as in Fig. 1 . Here $s$ stands for a characteristic specimen size and $P o$ denotes the value of $P$ at the macro scale. In Fig. 1 one can see the so-called positive size-effect, i.e. the increase of $P$ at $s$ tends to 0 . In order to describe this size-effect the scaling law was proposed, see [5], where it was illustrated in more details. The provided analysis of effective properties of plates and shells considering surface stresses shown that stiffness parameters follow exactly to this scaling law, 
see $[9,10]$. For example, the ratio of an effective bending stiffness to its classic counterpart coincides with the graph given in Fig. 1.
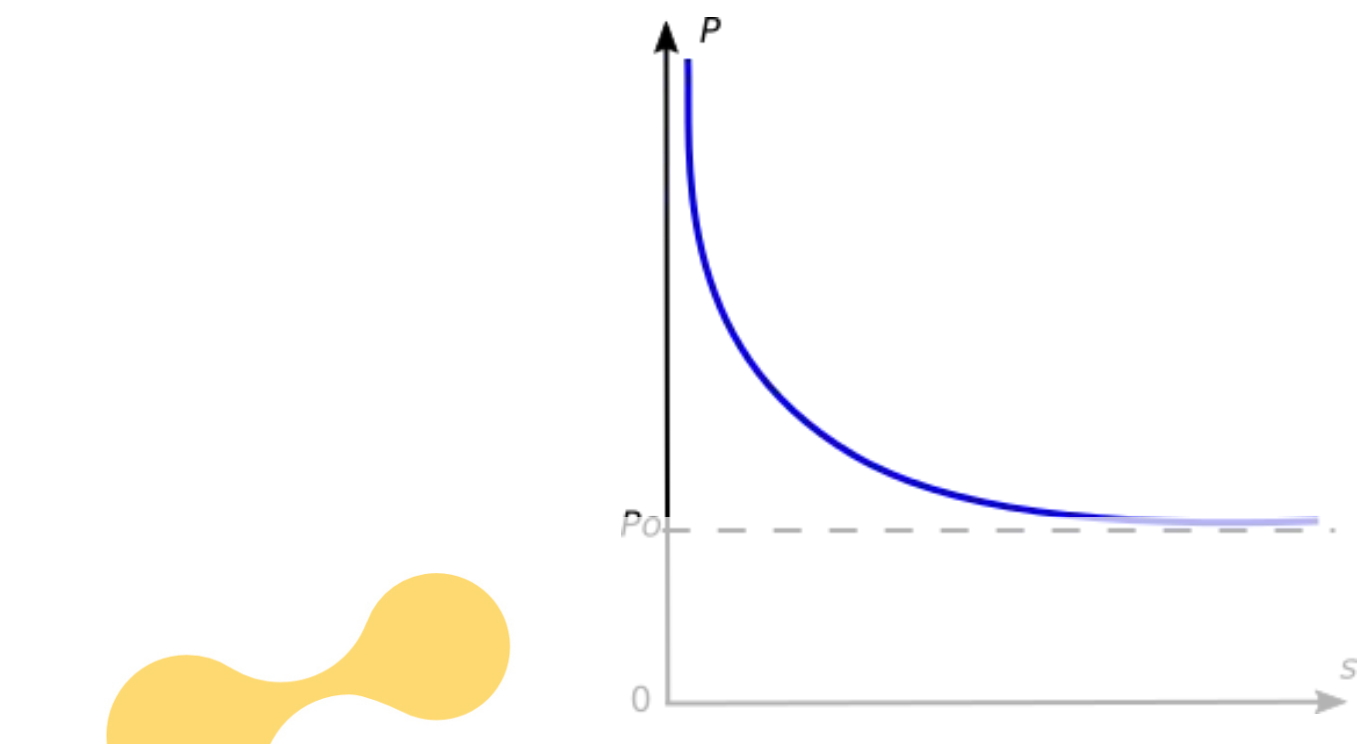

Figure 1: Positive size-effect: dependence of a material property $P$ on the length parameter $s$.

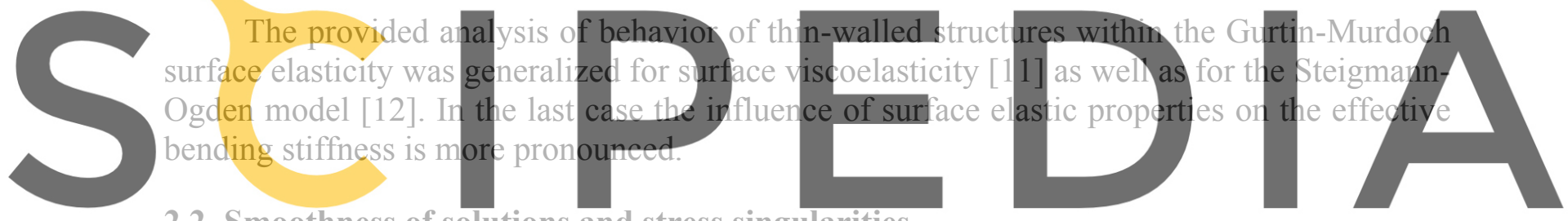

\subsection{Smoothmess of solutions and stress singularities}

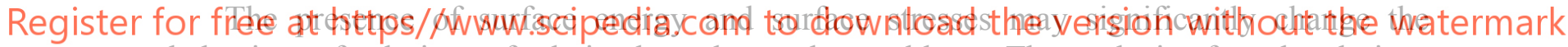
behaviour of solutions of relative boundary-value problems. The analysis of weak solutions within the linear Gurtin-Murdoch model was provided in [13, 14], whereas the similar analysis for the Steigmann-Ogden surface elasticity was given in [15]. Let us recall that by weak solution we mean a displacement field $u$ which satisfy the virtual work principle for all admissible functions

$$
\delta E \equiv B(u, v)=F(v)
$$

where $E=\iiint_{V} W d V$ is an energy functional, $\delta E$ is its first variation, which can be represented as a bilinear form $B(u, v), v=\delta u$, and $F(v)$ is a linear functional describing a work of external loads.

Let us recall that a weak solution in the classic elasticity has the following properties [16]

$$
u \in W^{1,2}(V),
$$

where $W^{m, 2}(V)$ and $L^{p}(V)$ are Sobolev's and Lebesgue functional spaces, Here we assumed that the body forces and surface traction have the following properties $f \in L^{6 / 5}(\mathrm{~V}), \mathbf{t} \in$ $L^{4 / 3}(S)$, respectively. Within the Gurtin-Murdoch surface elasticity we get that 


$$
u \in W^{1,2}(V) \cap W^{1,2}(S) \quad \mathrm{S}=\partial V .
$$

Considering eigen-oscillations of solids with surface stresses one can conclude that if the surface energy is positive we have always positive size-effect, i.e. stiffening [14].

For the Steigmann-Ogden model the weak solutions are even more smooth on the surface [15]. In particular, the normal component of displacements belongs to $W^{1,2}(V) \cap W^{2,2}(S)$. Moreover, for n-th-order surface elasticity a weak solution belongs to $W^{1,2}(V) \cap W^{n, 2}(S)$, see [17]. So for a smooth surface we have an essential improvement of the smoothness properties.

Another interesting discussion of the influence of surface stresses on a stress singularity near geometrical singularities such as a crack tip. In the literature various singularities (square root, logarithmic) or even an absence of any singularity were stated, see e.g. [18]. In [8] this problem was discussed in more details, where it was shown that the singularity level depend also on type of loads, i.e. depends on some symmetry properties.

\section{ANTI-PLANE SURFACE WAVES}

In Section 2 we briefly recall some modifications of properties of solutions as an effective properties and behavior of solutions of corresponding boundary-value problems. In addition to these phenomena we underline that the presence of surface energy and surfaces stresses may also result in new phenomena which are absent in the case of media without surface stresses. Among these phenomena let us mention here anti-plane surface waves. Surface waves are widely used in engineering, for example, for nondestructivg evaluation
defects.
3.1 Waves withim the limear Gurtin-Murdoch surface elasticity

The detailed analysis of the anti-plane propagation in media with surface stresses and in

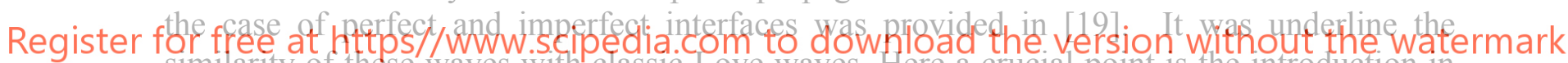
similarity of these waves with classic Love waves. Here a crucial point is the introduction in addition to (1) a surface kinetic energy given by

$$
K=1 / 2 m \dot{u},
$$

where $m$ is a surface mass density and the overdot stands for the derivative with respect to time. The similarity with Love waves can be also confirmed by discussion of transition conditions for so-called stiff interfaces, see $[8,20]$. The analysis of anti-plane waves was extended to the transverse waves on a cylindrical surface [20]. For a cylindrical surface this type of waves exists even without surface stresses, nevertheless their presence significantly changes a picture of wave propagation especially for short-length waves. More complex problem was considered in [21], where a roughness was taken into account. Let us note that in this case we have different characteristic length-scale parameters which essentially influence wave packet propagation along the surface.

\subsection{Comparison with the Toupin-Mindlin strain gradient elasticity}

Let us note that in the literature there is another model called strain gradient elasticity which can also address surface energy phenomena. The comparison of the Toupin-Mindlin 
strain gradient elasticity model with the Gurtin-Murdoch surface elasticity was provided in [22]. It was shown that the both models can capture the same phenomena of anti-plane surface waves. Nevertheless the difference in decay rate in depth direction is observed.

\subsection{Comparison with lattice dynamics}

Considering anti-plane surface waves at the nanoscale it seems quite natural to compare this behavior with lattice dynamics and find the continuum limit which can describe the surface elasticity. Such analysis was performed in [23] where for a square lattice an exact solution was obtained. In [23] we proposed also a scaling law similar to [5] which results in transition from lattice dynamics into the Gurtin-Murdoch surface elasticity. Using this discrete-continuum correspondence in [24] the analysis of wave refraction, transmission and leakage for a surface with linear defect was given.

The analysis of anti-plane surface waves was also performed for some generalizations of surface elasticity as $\mathrm{n}$-th-order surface elasticity $[17,25]$ or strongly anisotropic surface elasticity [26].

\section{CONCLUSIONS}

We have discussed of surface elasticity phenomena and their influence on the material behavior. The presence of surface energy and surface stresses is at least two-fold. First, surface elasticity change the effective (apparent) material properties as stiffness parameters. In particular, within the surface elasticity one can capture size-effects observed at the small scales. Considering the related boundary-value problems one properties of their solutions. For example, the stress singularity
of the surface stresses or the smoothness of solutions in the vicin surface stresses acts. Moreover, surface energy may also result in new phenomena such as forti-plane surface waves. The work was carried out with the linancial support or the Ministry of Science and Higher Education of the Russian Federation (task 0729-2020-0054).

\section{REFERENCES}

[1] Gurtin, M.E. and Murdoch, A.I., A continuum theory of elastic material surfaces. Arch. Ration. Mech. Analysis, (1975) 57(4):291-323.

[2] Gurtin, M.E. and Murdoch, A.I., Surface stress in solids. Int. J. Solid. Struct., (1978) 14(6):431-440.

[3] Steigmann, D.J. and Ogden, R.W. Plane deformations of elastic solids with intrinsic boundary elasticity. Proc. Roy. Soc. London. A, (1997) 453(1959):853-877.

[4] Steigmann, D.J. and Ogden, R.W. Elastic surface - substrate interactions. Proc. Roy. Soc. London. A, (1999) 455(1982):437-474.

[5] Duan, H.L., Wang, J. and Karihaloo, B.L. Theory of elasticity at the nanoscale. $A d v$. Appl. Mech., (2008) 42:1-68.

[6] Wang, J., Huang, Z., Duan, H., Yu, S., Feng, X., Wang, G., Zhang, W., and Wang, T. Surface stress effect in mechanics of nanostructured materials. Acta Mech. Solida Sin., (2011) 24:52-62. 
[7] Eremeyev, V.A. On effective properties of materials at the nano- and microscales considering surface effects. Acta Mech., (2016) 227:29-42.

[8] Gorbushin, N., Eremeyev, V.A., Mishuris, G. On stress singularity near the tip of a crack with surface stresses. Int. J. Engng. Sci., (2020) 146:103183.

[9] Altenbach, H., Eremeev, V.A. and Morozov, N.F. On equations of the linear theory of shells with surface stresses taken into account. Mech. Solids, (2010) 45(3):331-342.

[10] Altenbach, H. and Eremeyev, V.A. On the shell theory on the nanoscale with surface stresses. Int. J. Engng. Sci., (2011) 49(12):1294-1301.

[11] Altenbach, H., Eremeyev, V.A. and Morozov, N.F. Surface viscoelasticity and effective properties of thin-walled structures at the nanoscale. Int. J. Engng. Sci., (2012) 59:83-89.

[12] Eremeyev, V.A. and Wiczenbach, T. On effective bending stiffness of a laminate nanoplate considering Steigmann-Ogden surface elasticity. Appl. Sci. (2020), 10(21): 7402.

[13] Altenbach, H., Eremeyev, V.A. and Lebedev, L.P. On the existence of solution in the linear elasticity with surface stresses. ZAMM, (2010) 90(3):231-240.

[14] Altenbach, H., Eremeyev, V.A. and Lebedev, L.P. On the spectrum and stiffness of an elastic body with surface stresses. ZAMM, (2011) 91(9):699-710.

[15] Eremeyev, V.A. and Lebedev, L.P. Mathematical study of boundary-value problems within the framework of Steigmann-Ogden model of surface elasticity. Contin. Mech. Thermodyn., (2016) 28(1): 407-422.
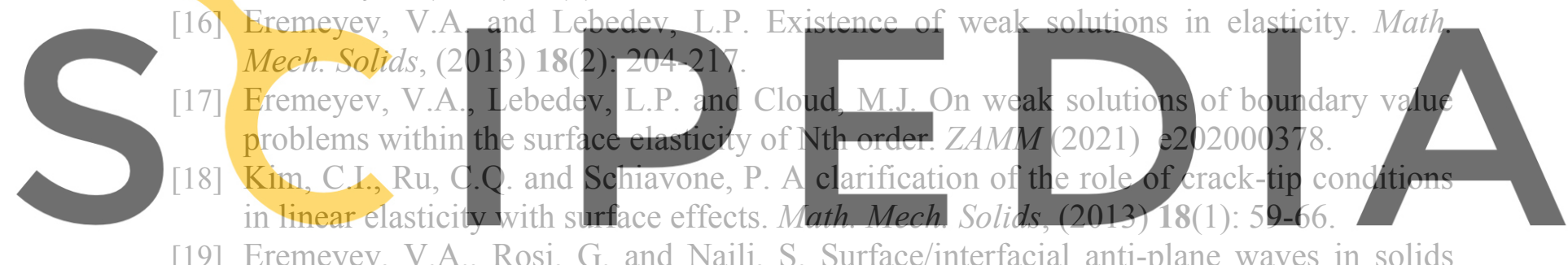

[19] Eremeyev, V.A., Rosi, G. and Naili, S. Surface/interfacial anti-plane waves in solids

Register for freetla

[20] Eremeyev, V.A., Rosi, G. and Naili, S. Transverse surface waves on a cylindrical surface with coating. Int. J. Engng. Sci. (2020) 147:103188.

[21] Mikhasev, G.I., Botogova, M.G. and Eremeyev, V.A. On the influence of a surface roughness on propagation of anti-plane short-length localized waves in a medium with surface coating. Int. J. Engng. Sci. (2021) 158:103428.

[22] Eremeyev, V.A., Rosi, G. and Naili, S. Comparison of anti-plane surface waves in strain-gradient materials and materials with surface stresses. Math. Mech. Solids, (2019) 24(8):2526-2535.

[23] Eremeyev, V.A. and Sharma, B.L. Anti-plane surface waves in media with surface structure: Discrete vs. continuum model. Int. J. Engng. Sci., (2019) 143:33-38.

[24] Sharma, B.L. and Eremeyev, V.A. Wave transmission across surface interfaces in lattice structures. Int. J. Engng. Sci., (2019) 145:103173.

[25] Eremeyev, V.A. Surface elasticity models: comparison through the condition of the antiplane surface wave propagation. In: State of the Art and Future Trends in Material Modeling (2019) (pp. 113-124). Springer, Cham.

[26] Eremeyev, V.A. Strongly anisotropic surface elasticity and antiplane surface waves. Phil. Trans. Roy. Soc. A, (2020) 378(2162):20190100. 